\title{
Management of acute cardiac tamponade following a transcatheter heart intervention.
}

\author{
Domenico Calcaterra ${ }^{1}$ \\ ${ }^{1}$ Bethesda Heart Hospital Baptist South Florida
}

October 8, 2020

\begin{abstract}
I read with interest the manuscript by Masroor and co-authors on the strategy of surgical repair for left atrial appendage perforation following implantation of Watchman device. The authors present the successful management of this complications and they comment on the preferred therapeutic strategy. This is a particular sensitive subject nowadays, since the widespread use and the constant growth of a variety of transcatheter cardiac interventions has inevitably increased the number of patients who are exposed to this type of complication.
\end{abstract}

Management of acute cardiac tamponade following a transcatheter heart intervention.

Domenico Calcaterra MD, PhD.

Bethesda Heart Hospital Baptist South Florida, Florida Atlantic University, Boynton Beach FL, USA.

This is an original article.

The author has not conflict of interest related to this work.

For Correspondence:

Domenico Calcaterra MD, PhD

Bethesda Heart Hospital

Baptist South Florida

2815 South Seacrest Blvd

Boynton Beach, FL 33435

domenicoc@baptisthealth.net

Ph. 561-374-5720

Word count: 937

I read with interest the manuscript by Masroor and co-authors on the strategy of surgical repair for left atrial appendage perforation following implantation of Watchman device. The authors present the successful management of this complications and they comment on the preferred therapeutic strategy. This is a particular sensitive subject nowadays, since the widespread use and the constant growth of a variety of transcatheter cardiac interventions has inevitably increased the number of patients who are exposed to this type of complication. 
My first comment to the authors is in regards to the choice of the surgical repair strategy. Cardiac surgeons have learned a lot from the experience of acute cardiac tamponade ${ }^{1}$ occurring with injuries secondary to pace-maker lead insertion or extraction, which more often involve damage to the right atrial wall ${ }^{2}$. This latter scenario is not dissimilar from the one presented in this manuscript, with the downsize that the rate of bleeding of a left atrial injury could be even more dangerous because of the higher left atrial pressure compared to the right atrium. In my experience, the safest way to approach a life threatening emergency of this type is to proceed with percutaneous pericardiocentesis in face of hemodynamic instability and plan for emergency operation if evidence of bleeding persists. The surgical approach I consider the safest is to establish extracorporeal circulation with peripheral arterial and venous femoral cannulation, which can be accomplished expeditiously either with percutaneous approach or surgical cutdown. The strong benefit offered by this strategy is allowing to establish cardiopulmonary bypass sooner, also avoiding to open the chest with ongoing active bleeding. Opening the chest without establishing peripheral preventive cardiopulmonary bypass may worsen acute blood loss which could cause cardiac arrest. Additionally, the presence of uncontrolled active bleeding can make cannulation maneuvers very cumbersome. Establishing cardiopulmonary bypass, allows reducing the amount of bleeding by 'decompressing' the heart and allows to facilitate exposure of the area of bleeding when the pericardium is opened following the sternotomy.

Second, I would discourage any attempt for a thoracoscopic approach to care for this complication. The author is correct mentioning the report of a thoracoscopic repair published in the literature. Nonetheless, the thoracoscopic approach in such a situation should not be recommended. In fact, choosing this strategy does not ensure safely obtaining control of the bleeding, since procedural exposure can be challenging. Also, being able to staple the left atrial appendage on a beating heart with ongoing bleeding may be unsuccessful or dangerous for causing damage to surrounding heart structure, the circumflex artery as an example. Furthermore, trying the thoracoscopic approach could definitely delay the delivery of the life-saving operation, which is obviously time-sensitive, and could unnecessarily jeopardize patient's survival. If this is a true emergency as it is, the standard of care should be focused on proceeding with the easiest and fastest approach, which by definition is a midline sternotomy.

Third, I am not convinced that opening the left atrium through the Waterston groove is necessarily the best approach ${ }^{3}$. In fact, the Watchman has the shape of a mushroom with a stalk and cap. Retrieving the device from inside the left atrium may be cumbersome and cause unnecessary damage to the base of the left atrial appendage, which could ultimately complicate the final repair. Therefore in this situation, I would favor to attempt removing the device from the outside of the heart first, after establishing cardioplegic arrest. Under these conditions, it is relatively safe to oversaw the appendage at its base with a double layer of 4-0 polypropylene suture.

Lastly, I am not sure I would recommend completing a Maze procedure in the contest of this emergent operation. According to the literature, complete exclusion of the left atrial appendage reduces the risk of stroke up to $90 \%$ in non-valvular atrial fibrillation and approximately $60 \%$ in valvular atrial fibrillation ${ }^{4}$. Therefore, I would be comfortable with minimizing cross-clamp and cardiopulmonary bypass time, aiming at saving the patient's life for the time being. Exposing the patient to increased risk of bleeding from performing the Maze procedure and facing the potential risk of causing new conduction abnormalities which may require permanent pacemaker placement could be, in my opinion, not justified in this contest. Additionally, is worth to mention that, as an alternative if needed, transcatheter ablation for treatment of the atrial fibrillation would also be a valid and suitable option on an elective base.

With the occasion I would like to thank the editor in Chief of the Journal of Cardiac Surgery for inviting my comments on this interesting manuscript.

References:

1. Spodick DH. Acute cardiac tamponade. N Engl J Med. 2003 Aug 14;349(7):684-90. doi: 10.1056/NEJMra022643. PMID: 12917306.

2. Jacheć W, Polewczyk A, Polewczyk M, Tomasik A, Janion M, Kutarski A. Risk Factors Predicting 
Complications of Transvenous Lead Extraction.Biomed Res Int . 2018;2018:8796704. Published 2018 Dec 18. doi:10.1155/2018/8796704

3. Jensen J, Thaler C, Saxena R, Calcaterra D, Sanchez J, Orlandi Q, Harris KM. Transesophageal Echocardiography to Diagnose Watchman Device Infection. CASE (Phila). 2020 Feb 18;4(3):189-194. doi: 10.1016/j.case.2020.01.008. PMID: 32577603; PMCID: PMC7303240.

4. Chatterjee S, Alexander JC, Pearson PJ, Feldman T. Left atrial appendage occlusion: lessons learned from surgical and transcatheter experiences. Ann Thorac Surg. 2011 Dec;92(6):2283-92. doi: 10.1016/j.athoracsur.2011.08.044. Epub 2011 Oct 25. PMID: 22029943. 